\title{
Influence of Foundation Rigidity on the Structural Response of Mat Foundation
}

\author{
Sami W. Tabsh (iD) and Magdi El-Emam \\ Civil Engineering Department, American University of Sharjah, Sharjah, UAE \\ Correspondence should be addressed to Sami W. Tabsh; stabsh@aus.edu
}

Received 1 March 2021; Revised 24 September 2021; Accepted 27 September 2021; Published 8 October 2021

Academic Editor: Qing-Xin Ren

Copyright (c) 2021 Sami W. Tabsh and Magdi El-Emam. This is an open access article distributed under the Creative Commons Attribution License, which permits unrestricted use, distribution, and reproduction in any medium, provided the original work is properly cited.

\begin{abstract}
A mat is a type of shallow foundation that is appropriate for structures supported on soil having relatively low bearing capacity or excessive settlement. Structural analysis of a mat foundation can be accomplished by either assuming the mat to be perfectly rigid or by considering the soil-structure interaction. This study researches the relationship between the mat-soil rigidity and structural response in terms of the soil bearing pressure, bending moment, and shear within the mat. To accomplish the objective of the study, 70 different mats are analyzed using a linearly elastic finite element approach. The variables that are considered in the analysis are the number of bays in each direction, center-to-center column spacing, mat thickness, panel aspect ratio, column cross section dimensions, soil modulus of subgrade reaction, and modulus of elasticity of concrete. A dimensionless mat rigidity measure was developed that determines whether a given mat can be reasonably analyzed by assuming it to be infinitely rigid. The developed rigidity factor takes into consideration all parameters that significantly affect the mat structural response. Results of the analysis indicate that there is strong correlation between the developed rigidity factor and critical soil bearing pressure and maximum internal bending moment within the mat. No correlation was observed between the mat rigidity and critical shear force. Relationships between the rigidity factor and the critical soil bearing pressures and bending moments, relative to the response of the infinitely rigid mat, are proposed. A parametric study is included to demonstrate the impact of the variables that affect the rigidity index on the response of the mat.
\end{abstract}

\section{Introduction}

Mats, also known as rafts, are a type of shallow foundation composed of a relatively thick concrete slab resting on earth and supporting moderate loads applied through columns and shear walls. They are often utilized when the shallow soil strata have inferior bearing capacity, resulting in strip or isolated footings covering more than $50-60 \%$ of the built area beneath the structure. In this situation, a raft is cheaper, easier to construct and offers a better water-tight structure compared to other foundation types. In addition, a mat reduces differential and total settlement and provides a bridge between local soils with different geotechnical properties that exist within the site. Furthermore, a mat foundation spreads the applied loads over the whole supported area and can be constructed with or without stiffening beams between the columns. An increase in the mat rigidity can improve the stability of the platform base, leading to enhancement in the serviceability of the supported structure due to reduction in settlement of the foundation.

The distribution and shape of the bearing soil pressure underneath a mat foundation depends on both soil stiffness and mat rigidity. When the relative soil-to-mat rigidity is high (e.g., thin mat on rock), a vertical load applied on the mat causes large bearing pressure over a narrow width located just below the load. For a foundation with moderate relative soil-to-mat rigidity ratio (e.g., medium thick mat on dense sand or stiff clay), the soil bearing pressure is reduced and distributed over a wider area within the vicinity of the 
load. In the case of a foundation having a high relative soilto-mat rigidity (e.g., thick mat on soft clay or medium dense sand), the soil bearing pressure is distributed somewhat linearly over the entire area of the foundation. These three cases are presented in Figure 1. From a structural point of view, the soil bearing pressure impacts the internal bending moment and shear force distribution and magnitude within mat.

Although there are several methods that have been suggested in the literature for the analysis of mat foundations, they can be grouped under two distinct categories: (1) rigid mat method and (2) flexible mat method. In the rigid method, the mat is assumed to behave as an infinitely rigid plate; hence, the soil bearing pressure is assumed to be linearly distributed under the mat, as shown in Figure 2(a). Due to this assumption, the type of soil and its properties do not affect the analysis, aside from satisfying the allowable bearing capacity. To satisfy the vertical force and bending moment equilibrium, the centroid of the soil pressure under the mat is assumed to coincide with the line of action of the resultant column loads above the mate. In this method, it is assumed that the flexural deflection does not affect the soil bearing pressure distribution under the mat. For the structural analysis, the mat is divided into a number of strips running along the two principal axes and loaded by a line of columns from the top and resisted by the soil pressure from the bottom. These strips are analyzed in a way similar to that used for the analysis of combined footings. While the rigid mat method is simple to use, experience has shown that it often overestimates both the mat thickness and steel reinforcement [1]. In addition, the method lacks the balance in equilibrium equations used for constructing shear force and bending moment diagrams for each strip, leading to erroneous results. Nowadays, applicability of this method is limited for rafts having small plan areas and simple geometries, as well as for preliminary sizing of complex ones.

In the flexible mat method, the foundation is assumed to be flexible, and the soil behavior is incorporated in the model, resulting in nonlinear distribution of soil bearing pressure underneath the foundation, as shown in Figure 2(b). In this case, differential settlements are expected to be relatively larger compared to the conventional rigid method; however, bending moments and shear forces within the mat resulting from the flexible approach are anticipated to be comparatively smaller. Nowadays, the finite element method is usually employed as a tool to execute the solution of the flexible mate approach. Modeling the mat by this method starts by subdividing the concrete slab into small rectangular or triangular plate or shell elements, with finer mesh introduced at the regions near the columns, re-entrant corners, openings, and regions of discontinuity. If desired, these elements can also account for shear deformation, which is important when the span-to-depth ratio of the mat is small. The soil could be represented simply by springs lumped at the nodes (Winkler model) or by half-space medium (continuum model). In the former approach, the stiffness of the springs is determined based on the tributary area associated with each node. If the soil is modeled as a half-space medium, its behavior can be calculated by dividing the surface into regions and the elements within a specific region are assigned suitable constitutive model that best describe the soil behavior at that location. The continuum model gives more accurate results of the stresses and distortions inside the soil mass than the Winkler model, but requires more modeling skill, as well as computational effort and time. Either way, the soil characteristics model can be defined as linear or nonlinear, and can be coupled or decoupled from each other.

\section{Problem Statement}

Accuracy of the rigid mat approach is greatly affected by the relative rigidity of the soil to that of the mat. Also, the stiffness of the supported superstructure (bridge vs. high-rise building) may have some effect on the mat behavior, although structural codes and specifications do not always address this issue. Selection of one analysis method over the other depends on the mat's relative rigidity with respect to the soil. For example, a relatively stiff mat can be properly analyzed using the rigid method that assumes the soil pressure to be linear, while a relatively flexible mat requires an advanced method of analysis that considers the soilstructure interaction. Different equations have been proposed in some design standards, such as the ACI Committee Report 336 [2] and the DIN-code 4018 [3], to calculate the mat rigidity factor. However, the available methods address the rigidity of the mat along two independent directions located within the plan area of the mat while ignoring the two-way bending action of the foundation. A problem arises if the rigidity of the mat along one direction is much different from the other direction. Hence, there is a need to derive a single rigidity factor that accounts for the stiffness of the mat as a unit.

\section{Literature Review}

Among the early published research on the analysis of mat foundation is the work of Ueshita and Meyerhof [4], who used experimental and theoretical methods to investigate the deflection of a circular footing supported on multilayered soil profile. They concluded that the deflection-pressure relationship can be better represented by an exponential model than a straight line. In the same track, Milovic and Tournier [5] provided solutions for soil bearing pressure and settlement produced by a uniformly loaded mat having a rectangular shape. They produced a dimensionless coefficient that could be used to determine the critical stress and displacement under a rigid rectangular mat. Tabakman and Hadjian [6] investigated the effect of circular- and rectangular-shaped foundation on the seismic response of nuclear power plant structures. The study confirmed that the practical thicknesses employed in such structures can be assumed rigid for the sake of computing impedance coefficients for soil-structure interaction analysis.

Ramanathan [7] and Ramanathan and Pujar [8] reviewed the available methods that consider the effect of superstructure rigidity on the structural response of rafts and used the method of consistent deformations to develop a 


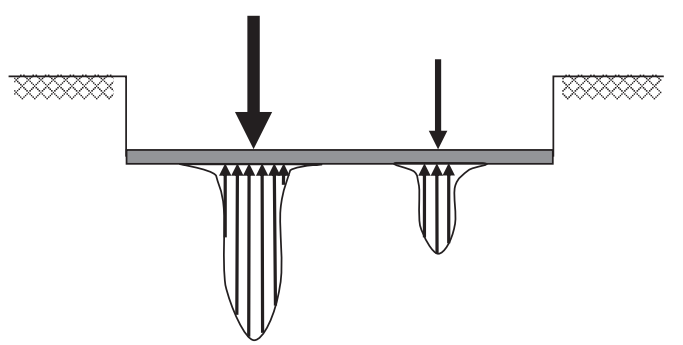

(a)

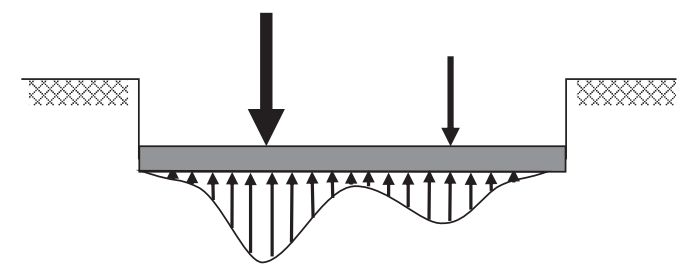

(b)

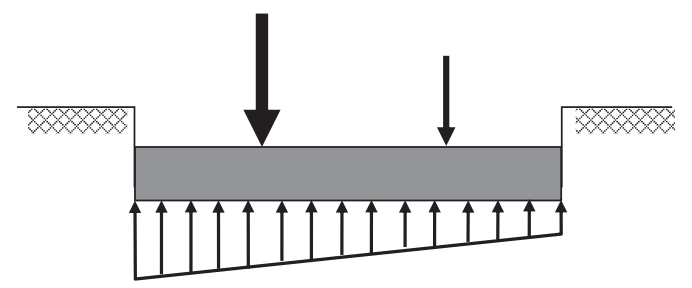

(c)

Figure 1: Effect of soil and mat stiffness on soil bearing pressure distribution. (a) Thin mat on rock. (b) Moderately thick mat on stiff soil. (c) Thick mat on soft soil.

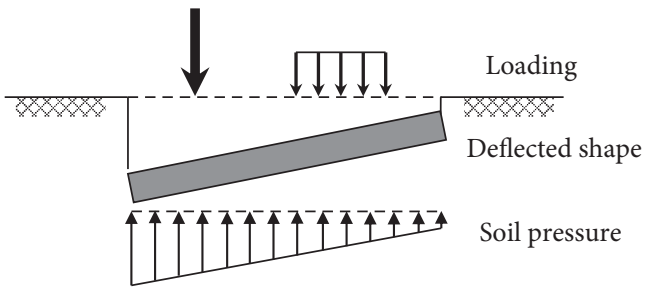

(a)

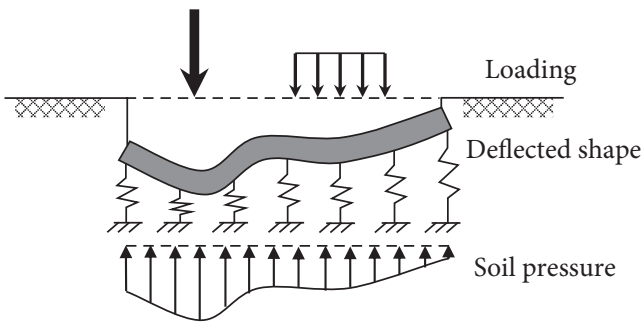

(b)

FIgUre 2: Soil pressure distribution in the rigid and flexible methods of analysis. (a) Rigid foundation. (b) Flexible foundation.

"relative stiffness index" for rectangular rafts. The study addressed rafts having different length-to-width ratios and various loading conditions with consideration of uplift. They found out that the combined rigidity of superstructure, raft, and soil have significant effects on the bending moment, settlement, and contact bearing pressure of the mat foundation. A similar study has been conducted by Mehrotra et al. [9], who introduced an approximate procedure based on the stiffness method that proven to give more accurate results than the traditional rigid method of analysis and leads to $25 \%$ savings in the raft steel and concrete material. Waas and Riggs [10] and Riggs and Waas [11] considered the effect of flexibility of a circular-base mat on the seismic response of an axisymmetric power plant reactor building loaded through two rigid concentric walls. They concluded that the behavior of the mat is dependent on the raft flexibility, load distribution, and frequency of excitation. For in-phase load distributions, the response of the flexible raft is similar to that of a rigid one at low frequencies but diverges at high frequencies. However, the authors found out that mat rigidity strongly affects the structural deformation modes.

A simple method that considers the interaction between the superstructure, raft, and soil was proposed by Yao and
Zhang [12]. The method addresses variations in superstructure rigidity, raft thickness, and elastic modulus of the supporting soil. They concluded that the variation of the mat relative rigidity with respect to the soil significantly affects the foundation differential settlement and forces in members of the superstructure. In addition, differences in the soil elastic modulus significantly affect the total settlement of the foundation and slightly impact both the differential settlement and forces in the structure members. A numerical approach is utilized by Chow [13] to determine the deformation of rigid foundations of arbitrary shape on soil. For a foundation on single-layer soil medium, the flexibility coefficients were given analytically based on Boussinesq's equation, whereas for multilayer soil medium, the same coefficients were obtained from a finite element analysis. Comparison of settlement results with solutions obtained using the integral transform technique showed good agreement between the two approaches.

Zilch [14] proposed a structural analysis method that considers the interaction of a building superstructure and its foundation through iteration. Application of the analysis procedure was illustrated on the foundation of a parking garage. It was found that for stiff structures, differential 
settlements can cause large internal forces in the partitions of the superstructure. In addition, Zilch concluded that modeling the soil as continuum or elastic half-space produces realistic deformation behavior. Horikoshi and Randolph [15] examined the effect of raft rigidity on the settlement of the foundation and developed a raft-soil stiffness ratio applicable to rectangular rafts subjected to uniform load. The ratio allows for direct evaluation of the critical differential settlement and maximum bending moments in the raft. In their method, they assumed a rigid raft and calculated the average settlement and maximum bending moment for this raft. Then, the differential settlement and the actual central bending moment are calculated as portions of the average settlement and maximum bending moment of the rigid raft. Other methods of raft analysis can be found in the book by Hemsley [16], who covered topics related to plates of various shapes and boundary conditions supported on Winkler springs and subjected to various types of loading.

Sutradhar [17] conducted a parametric study with the aid of the finite element method for the purpose of improving the design of mat foundation. He found out that by reducing the mat thickness away from the columns by up to $35 \%$, a more economical mat can be obtained without compromising the structural capacity of the foundation. In addition, results showed that in spite of discontinuity among springs, Winkler's foundation model gives very good idealization of soil, thus proving it to be a highly recommended model for design of foundations. He concluded that the finite element method yields substantial economy over the rigid method of analysis. Results of Sutradhar [17] were in agreement with the experimental results of Chown and Crilly [18], who conducted a full-scale raft foundation test. Gong et al. [19] used model tests to investigate the behavior of large mats supporting tall buildings. Findings of the tests demonstrated that a mat can be considered rigid if the mat thickness is larger or equal to one-sixth the spacing between columns, resulting in a near-linear soil bearing pressure distribution. Edgers et al. [20] examined the effects of soil-structure interaction of a tall building carried by a mat foundation considering different column support conditions. They found out that ignoring the foundation and subsoil rigidity by assuming a pin support condition at the columns base can underestimate the bending moments in the superstructure frame members by a factor of 2 to 3 . Modeling of soil with a nonlinear elastic-plastic relationship leads to more uniformly distributed soil bearing pressure and greater vertical displacements than a corresponding analysis utilizing elastic relations.

Farag et al. [21] conducted field tests and finite element analyses to determine the impact of raft thickness, superstructure rigidity, and soil properties on the soil-structure interaction. Data obtained from the field and numerical studies were used as input to an artificial neural network module to allow for calculating the redistributed column loads on the raft. Results of the study showed the importance of the interaction on differential settlement, especially for rafts supporting flexible superstructures. Thangaraj and Ilamparuthi [22] studied the raft foundation behavior of a typical 5-story structure with 3 bays in each direction on linearly elastic soil. They proposed two relative stiffness factors: one between the superstructure and the raft and another between raft and ground. They concluded that the settlements are nearly independent of the two stiffness factors. The settlements obtained with the interactive and noninteractive methods are quite similar for a foundation possessing low stiffness, and the bending moments within the superstructure elements are just about the same using interactive and noninteractive methods. Arapakou and Papadopoulos [23] researched the factors that affect differential settlements of 2-dimensional framed structures supported on spread footings, flexible mats, and rigid mats on linearly elastic or elastoplastic soil using the finite element method. Findings of the study showed that extremely flexible rafts can lead to similar results to isolated footings, magnitude of the loads is not influenced by the soil-structure interaction for very flexible frame or very rigid raft, and the bending moment within the raft is very sensitive to the rigidity of the foundation. Omer and Arbabi [24] evaluated the applicability of various methods used for the analysis of rafts on layered strata. They found out that deformations obtained by finite element and finite difference methods are close when the raft is flexible. Also, the soil bearing pressure computed from elastic methods did not agree with results from the finite element analysis when bedrock existed within depths shallower than 4 times the raft plan dimension, which indicates the importance of the soil modulus of subgrade reaction. Latka and Repelewicz [25] examined the influence of the effective stiffness of a raft foundation on Winkler's springs on the internal force distribution within the foundation. It was found that the presence of shear walls causes the raft to act together with the floor as a rigid box, causing favorable internal force distribution in the entire system. More recently and within the same context, Venkatesh and Deshpande [26] studied the effect of the soil-structure interaction under seismic loading on the response of 8-story reinforced concrete building on raft with consideration of different soils and seismic zones. As expected, the study showed that the natural period of vibration rises with soil flexibility, forces in the columns and beams increase for buildings on fixed supports, and the structural response becomes more critical with softening of the soil behavior and presence of higher seismic zone.

\section{Objectives and Scope}

The effort and time required to implement the flexible mat method of analysis are much more than those needed for the rigid mat method of analysis. This is because the former method is based on a higher-order analysis, such as the finite element or difference method, which often necessitates the use of software. Therefore, there is a need for developing a measure that can provide guidance on whether the simple rigid mat method of analysis is suitable or not for a specific mat and soil properties. Hence, the objective of this study is to derive a rigidity factor that accounts for the relative stiffness of the mat to the soil, which can be used to enable the foundation engineer to select an appropriate method of 
analysis for a given mat foundation. The proposed rigidity factor should rectify the deficiencies in the equations currently used to calculate the relative rigidity factors by treating the rigidity of the mat as a unit rather than considering its rigidity along two independent directions. Another objective for the research is to provide corrections factors to the soil bearing pressures and internal load effects within a mat analyzed by the rigid method of analysis.

The approach that is followed to achieve the objectives of the study consists in carrying out finite element analyses on mats having regularly and irregularly shaped plan geometries with different material properties, column spacing, number of bays, column sizes, mat thicknesses, and soil properties. This study builds on the previously published research of the two authors $[27,28]$ on the sensitivity of the response of mat foundations to changes in the design variables.

\section{Methodology}

All mats in this study are analyzed within the elastic range by the finite element method using the SAFE software $[29,30]$. The concrete mat is modeled by thick-shell elements and the soil by elastic springs lumped at the nodes, as shown in Figure 3. The thick-plate modeling in SAFE accounts for shear deformations in accordance with the Mindlin-Reissner approach, which is appropriate when the mat thickness-to-spacing between columns ratio is not large. Supporting the mat on discrete elastic springs is convenient because such an approach requires just one soil parameter, which is the modulus of subgrade reaction. The software is capable of modeling soil supports with zero tension to allow for an analysis that includes uplift. The stiffness of the elastic springs is adjusted whenever the mesh size changes. Rigid zones can be assigned within the mat to prevent slab rotational deformations at column locations. The boundary condition at the edges of the mat is not restrained against displacement and rotation to replicate the real situation. The loads are applied through the columns based on their tributary areas, resulting in concentrically loaded mats. For example, in a symmetrical mat with equal column spacing, the loads on the corner and edge columns are equal to onequarter and one-half of the load on the interior column, respectively. Self-weight and live load on the mat surface are ignored in the analysis because they are directly supported by the soil under the mat; hence, their contribution to the bending moment and shear within the mat is minimal.

The mesh size of the finite element model is chosen after carrying out a convergence analysis of a reference mat for which the geometry, material, and soil properties are shown in Table 1 . In the analysis, 4 mesh sizes varying between $0.25 \mathrm{~m}$ and $2 \mathrm{~m}$ were considered, as shown in Figure 4, leading to a suitable mesh size of $0.5 \mathrm{~m} \times 0.5 \mathrm{~m}$ that balances the computational time against accuracy of the results.

The variables that are considered in the analysis are the number of bays, column spacing, mat thickness, soil modulus of subgrade reaction, and column cross section dimensions. Note that mats having different number of bays along the length and width affect the aspect ratio of the

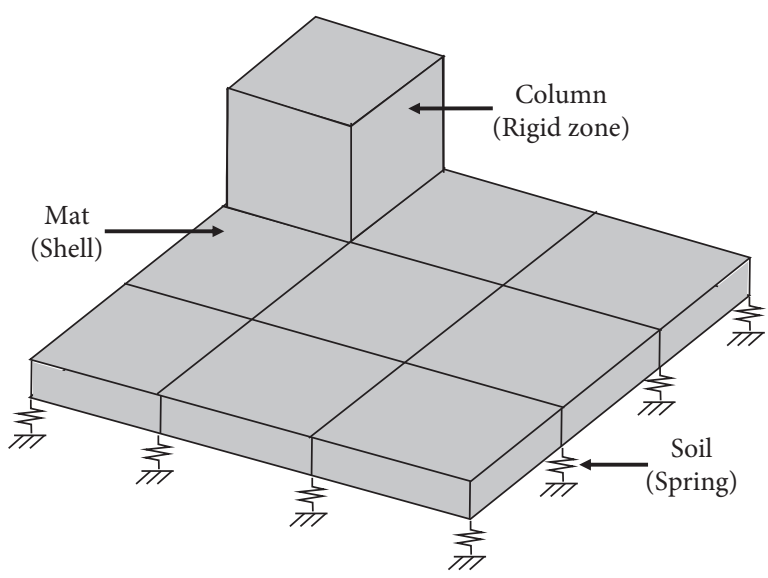

FIGURE 3: Details of the finite element model in SAFE.

TABle 1: Parameters of the reference mat used in the convergence analysis study.

\begin{tabular}{lc}
\hline Design parameter & Value \\
\hline Concrete modulus of elasticity $\left(\mathrm{KN} / \mathrm{m}^{2}\right)$ & $25,000,000$ \\
Modulus of subgrade reaction $\left(\mathrm{KN} / \mathrm{m}^{3}\right)$ & 50,000 \\
Concrete Poisson's ratio & 0.2 \\
Spacing between columns along width $(\mathrm{m})$ & 6 \\
Spacing between columns along length $(\mathrm{m})$ & 6 \\
Mat thickness $(\mathrm{m})$ & 1 \\
Column size $(\mathrm{m} \times \mathrm{m})$ & $0.3 \times 0.3$ \\
Corner column load $(\mathrm{kN})$ & 100 \\
Edge column load $(\mathrm{kN})$ & 200 \\
Interior column load $(\mathrm{kN})$ & 400 \\
\hline
\end{tabular}

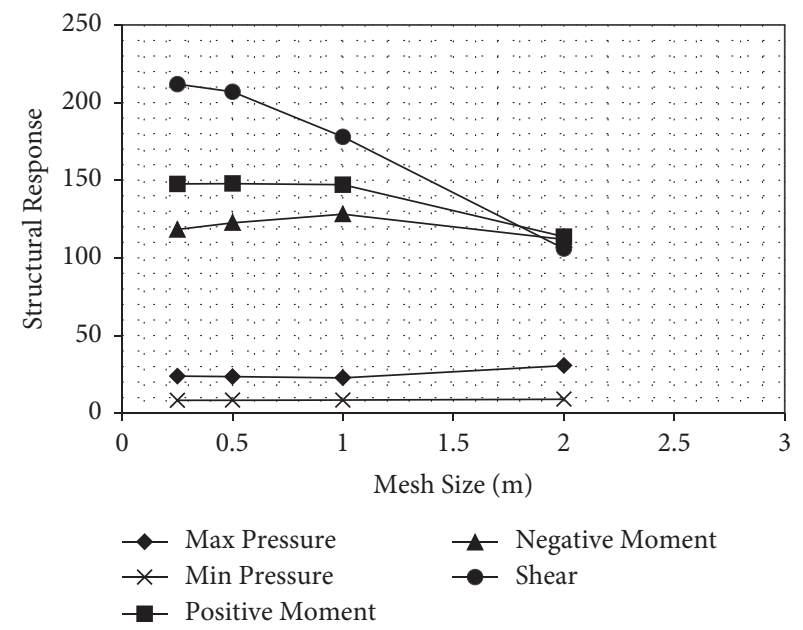

FIgURE 4: Results of the convergence analysis based on mesh size.

whole mat, while column spacing impacts the aspect ratio of the individual panels. In addition to considering regularly shaped mats, irregular mats with openings and with reentrant corners are also accounted for in the study, as shown in Figure 5. In total, 70 different cases are analyzed, in which the range of the number of bays is $2-5$, center-to-center column spacing is $3-12 \mathrm{~m}$, mat thickness is $0.5-5 \mathrm{~m}$, soil 

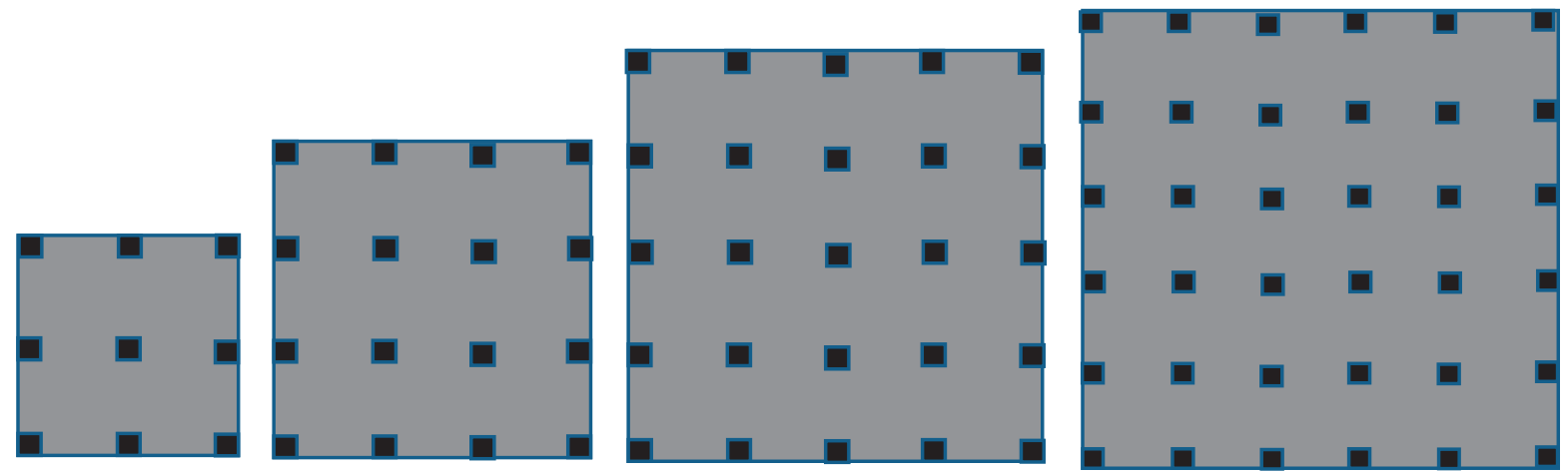

(a)

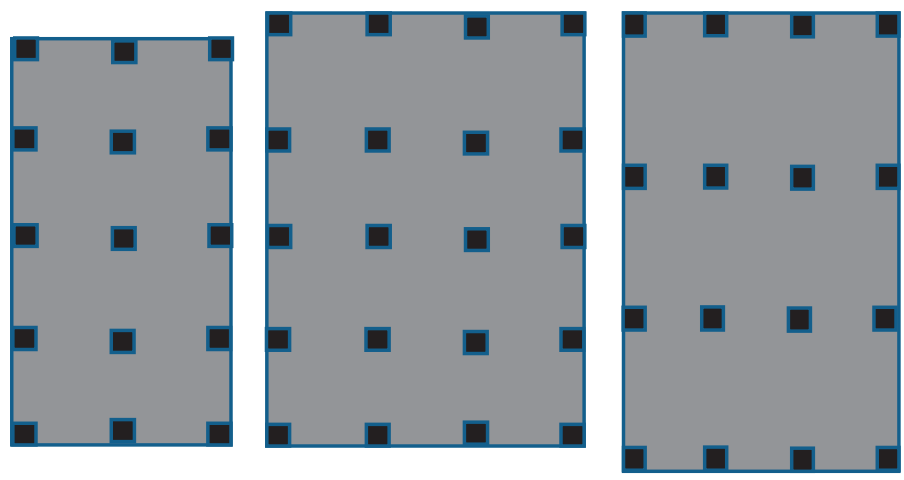

(b)

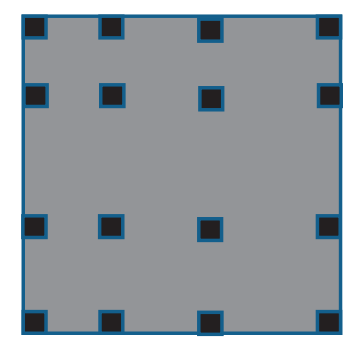

(c)
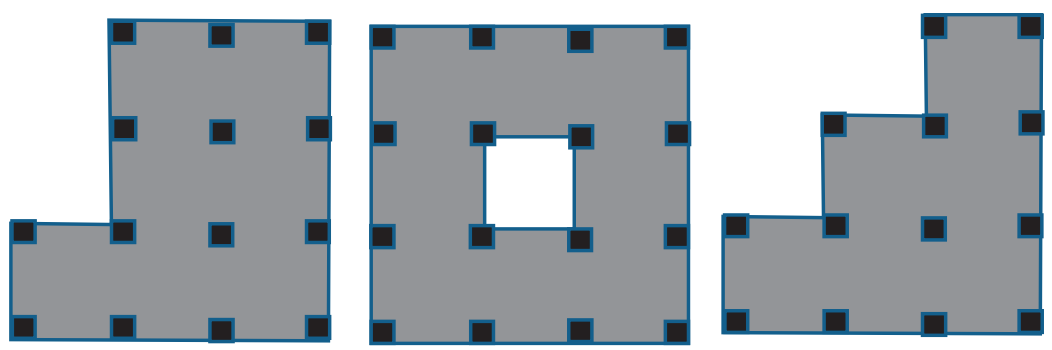

(d)

FIGURE 5: Geometry of the mats considered in the analysis. (a) Equal number of bays along length and width. (b) Unequal number of bays along length and width. (c) Rectangular panels. (d) Irregular geometries.

modulus of subgrade reaction is $10-400, \mathrm{MN} / \mathrm{m}^{3}$, panel aspect ratio is $0.5-1.0$, column side dimension is $0.3-1 \mathrm{~m}$, and modulus of elasticity of the concrete is $20-40 \mathrm{GPa}$. The Poisson ratio of the uncracked concrete used in the mat foundation is taken as 0.2 , since such a variable varies within a very narrow range.

In order to determine an effective relative rigidity factor for mats, the structural behavior must be judged on a comparison between a flexible mat and its infinitely rigid counterpart. Therefore, each mat in this study is analyzed twice, one with its actual thickness and another time with a $100 \mathrm{~m}$ thickness. The $100 \mathrm{~m}$ thickness for the designated infinitely rigid mat was chosen after examining the minimum and maximum soil bearing pressure under the mat for a variety of large thicknesses. Note that for an infinitely rigidly symmetrical mat, the minimum and maximum soil bearing pressure are equal to each other.

\section{Results}

From the available literature on foundation rigidity, it was found that the rigidity factor must account for the mat material and geometric properties, as well as the soil properties. The usual form of a rigidity measure contains the variables that are related to stiffness of the mat in the numerator and the same for the soil in the denominator. For example, the German DIN-code 4018 [3] and the ACI 336 committee report [2] have adopted the early work of Meyerhoff [31] to define a dimensionless foundation system stiffness, $K_{r}$, based on a strip having a unit width. This factor allows for distinguishing between flexible and stiff foundation behavior as follows:

$$
K_{r}=\left(\frac{1}{12}\right)\left(\frac{E_{c}}{E_{s}}\right)\left(\frac{t}{L}\right)^{3},
$$


where $E_{c}$ is the modulus of elasticity of the foundation material, $E_{s}$ is the modulus of elasticity of the supporting soil, $t$ is the foundation thickness, and $L$ is the foundation length. Since the modulus of subgrade reaction, $k_{s}$, is the only soil parameter accounted for in the flexible method of analysis that employs spring supports, the authors propose modifying the expression of the mat rigidity by considering the relationship between the modulus of elasticity and the subgrade reaction of the soil $[32,33]$ :

$$
E_{s}=k_{s} L\left(1-\mu_{s}^{2}\right),
$$

where $\mu_{s}$ is the Poisson ratio of the soil.

By substituting the expression of $E_{s}$ from (2) in the expression of $K_{r}$ in (1), eliminating the constant from the denominator, and using the maximum clear distance between columns along the two principle axes of the mat, a relative mat rigidity factor $K_{r}^{\prime}$ is obtained:

$$
K_{r}^{\prime}=\frac{E_{c} t^{3}}{k_{s}\left(1-\mu_{s}^{2}\right)(L-l)^{2}(B-b)^{2}},
$$

in which $L$ is the maximum center-to-center distance between the columns along the length of the mat, $B$ is the maximum center-to-center distance between the columns along the width of the mat, $l$ is the column cross section dimension along the length of the mat, and $b$ is the column cross section dimension along the width of the mat. During the development of the proposed rigidity factor, the authors tried using the minimum and average spacing between columns and also the total mat dimensions, but the maximum spacing between columns was found to correlate better with the structural response. The above expression of $K_{r}^{\prime}$ in (3) for mat foundations is the same as that proposed by the first author for estimating the rigidity of spread footings [34], but with different definitions of $L$ and $B$.

In presenting the relationships between the mat response and the proposed rigidity factor, the Poisson ratio of the soil is needed in the equation of the rigidity. Such a parameter is not required in the flexible method of analysis that utilizes discrete elastic springs to model the soil since the only soil parameter used is the modulus of subgrade reaction. In the subsequent analysis, a value of $\mu_{s}$ equal to 0.3 is used for calculating $K_{r}^{\prime}$, which represents an average value for most soil types. Deviations in the Poisson ratio of the soil towards extreme values of 0.2 (loose sand or soft clay) and 0.4 (dense sand or stiff clay) do not impact the magnitude of the rigidity factor by more than $8 \%$.

Figure 6 presents the variation of the maximum and minimum soil pressure ratios under the mat foundation versus the relative rigidity factor. The extreme pressure ratio is computed by dividing the maximum and minimum observed soil pressures of a given mat by the uniform pressure of an infinitely thick mat having the same design parameters. The corresponding maximum bending moments and shear force per unit strip ratios of the considered mats are shown in Figures 7 and 8, respectively.

With regard to the results concerning soil bearing pressure, presented in Figure 6, the finite element analysis

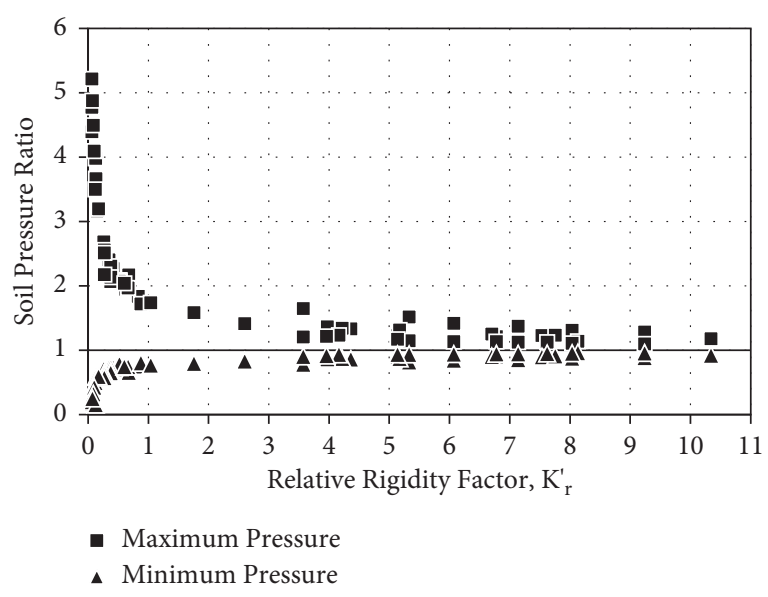

Figure 6: Variation of soil pressure ratio with mat rigidity.

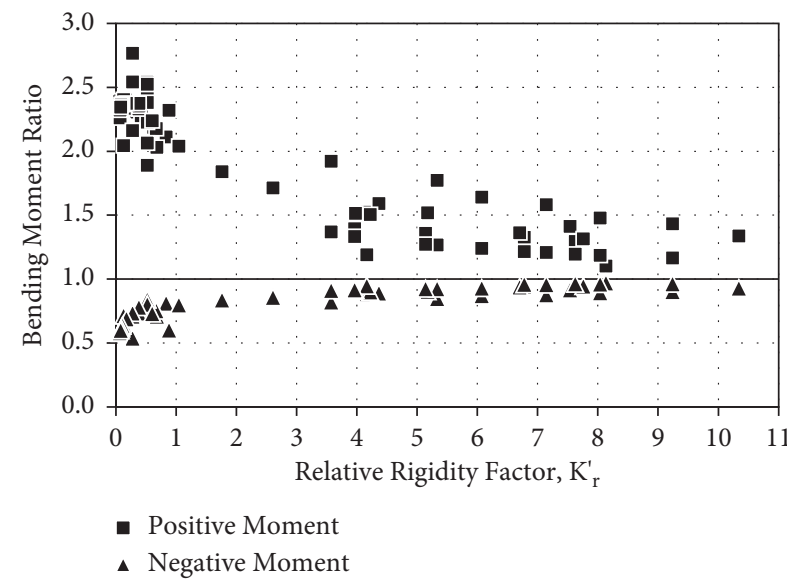

Figure 7: Variation of bending moment ratio with mat rigidity.

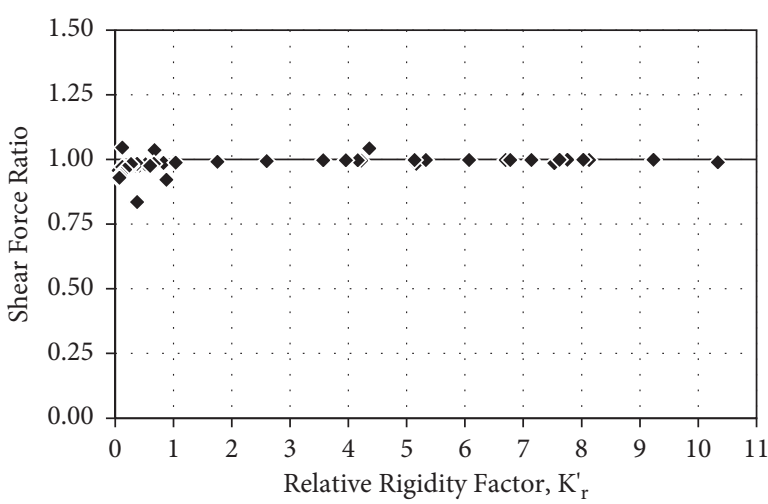

FIgURE 8: Variation of shear force ratio with mat rigidity.

showed that the maximum soil pressure often occurs below the corner columns due to lack of continuity of the raft at that location, which results in larger deflection under these columns. On the other hand, the minimum soil pressure was frequently noticed within the central region of the raft due to the relatively larger deflection along the perimeter, leading to upward bulging inside the central area. As expected, Figure 6 indicates that as the mat rigidity increases, the maximum soil 
pressure ratio decreases while the minimum soil pressure ratio increases, approaching a state of uniform pressure distribution at a very large value of rigidity. The maximum soil pressure ratio exhibits a sharp decrease and the minimum soil pressure ratio displays a sharp increase when the rigidity rises from 0 to 1.0. Thereafter, the two critical soil pressure ratios start converging to 1.0. Based on the obtained results, it can be deduced that mats having rigidity factor less than 1.0 can be considered flexible and those having rigidity factor more than 1.0 can be considered rigid. Practically, investigating a flexible mat using the rigid mat method of analysis will underestimate the critical stresses within the mat, thus compromising the factor of safety.

Figure 7 presents the maximum positive (compression at top) and negative (tension at top) bending moment per unit strip ratios versus the proposed rigidity factor. The bending moment is the result of integrating the shear force over a part of the mat area. The scatter in the positive and negative moment values for flexible mats with $K_{r}^{\prime}<1$ is primarily due to the scatter in the critical shear values, discussed in the next paragraph. Moreover, the dispersion of the positive moment values for $K_{r}^{\prime}>1$ is due to the sensitivity of the positive moment to changes in the soil bearing pressure distribution caused by the abrupt change in the mat stiffness in the vicinity of the columns and the various mat geometries considered in the study. Figure 7 shows that, in general, the finite element analysis indicates that the critical negative moment occurs midway between the columns located at the borderline between the corner and nearby edge panels, whereas the critical positive moment occurs under the interior column of the corner panel. The variation in bending moment with changes in mat rigidity is mainly due to the change in soil bearing pressure distribution. In the case of flexible mats, the soil pressure under the columns is large and concentrated over small areas, while in the case of stiff mats the pressure is small in magnitude and spread out. For flexible mats $\left(K_{r}^{\prime}<1\right)$, the maximum positive moment ratio is much larger than the corresponding maximum negative bending moment ratio, and both are highly sensitive to the rigidity of the mat. The results also show that the maximum positive moment ratio becomes smaller and maximum negative moment ratio becomes larger with an increase in the mat rigidity factor. This result is not surprising because as the mat becomes rigid, the soil bearing pressure distribution changes from a localized state under the columns into a more uniform condition underneath the whole mat. Presence of significant uniform soil pressure between the columns is responsible for the increase in the negative moment between the columns and decrease in the positive moment under the columns.

The influence of mat rigidity on the maximum shear force ratio at the face of the critical column is presented in Figure 8. Note that the shear force is the result of integrating the soil bearing pressure over a part of the mat area. The scatter of the shear values for flexible mats with $K_{r}^{\prime}<1$ is mainly due to the abrupt change in stiffness of the mat in the vicinity of the columns. The results indicate that the maximum shear within the mat for $K_{r}^{\prime}>1$ is independent of the mat rigidity because the critical shear within the mat is directly related to the integral of the soil bearing pressure over the influence area under the mat, regardless of its distribution, which changes with the mat rigidity.

Trend lines are fitted to the obtained finite element results to relate the relative soil pressure and internal load effects to the proposed mat rigidity factor. It was found that the best and simplest correlations to consider in the study are power functions, in the form of $y=a x^{b}$. The trend lines for the critical maximum and minimum soil pressure ratios, $(S P R)_{\text {max }}$ and $(S P R)_{\text {min }}$, have coefficients of determination equal to 0.933 and 0.607 , respectively, and are valid for $K_{r}^{\prime} \leq 10$. The relatively low value of the coefficient of determination for the equation that relates the minimum soil bearing pressure to mat rigidity is due to the small value of the minimum soil pressure which causes sensitivity to minor changes and also the inability of the chosen power function to capture the rapid drop in the $y$-coordinates within the small $x$-values. They are presented in Figure 9 and shown below as a function of the mat rigidity factor, $K_{r}^{\prime}$ :

$$
\begin{aligned}
& (\mathrm{SPR})_{\max }=1.929\left(K_{r}^{\prime}\right)^{-0.262,} \\
& (\mathrm{SPR})_{\min }=0.684\left(K_{r}^{\prime}\right)^{0.1974} .
\end{aligned}
$$

The corresponding trend lines for the critical maximum positive and negative bending moment ratios, $\left(B M R^{+}\right)_{\max }$ and $\left(B M R^{-}\right)_{\max }$, have coefficients of determination equal to 0.792 and 0.830 , respectively, and are applicable for $K_{r}^{\prime} \leq 10$. They are presented in Figure 10 and shown below:

$$
\begin{aligned}
& \left(B M R^{+}\right)_{\max }=1.869\left(K_{r}^{\prime}\right)^{-0.152}, \\
& \left(B M R^{-}\right)_{\min }=0.789\left(K_{r}^{\prime}\right)^{0.0873} .
\end{aligned}
$$

No curve fitting is presented for the shear results since the finite element results indicated that such a response is independent of the mat rigidity. The developed power functions, presented in equations (4) to (7), can be used to estimate the critical soil bearing pressures and bending moments in a flexible mat from the corresponding results of an equivalent rigid mat having the same plan geometry and loading.

\section{Parametric Study}

The developed trend lines in equations (4)-(7), together with the proposed expression of the mat rigidity in (3), can be used to gain insight into the sensitivity of a given mat to changes in the geometric and material properties. This approach can be illustrated by considering a square symmetrical mat carrying 12 columns, having a thickness of $1.2 \mathrm{~m}$, modulus of elasticity of concrete of $25 \times 10^{6} \mathrm{kPa}$, and supported on soil with a modulus of subgrade reaction equal to $50,000 \mathrm{kN} / \mathrm{m}^{3}$ and Poisson's ratio of 0.25 . The uniform center-to-center spacing between the columns is $6 \mathrm{~m}$ and the cross section of the columns is $0.50 \mathrm{~m}$ by $0.50 \mathrm{~m}$, as shown in Figure 11. The reference mat shown in Figure 10 has a rigidity factor $K_{r}=1.0$, according to equation 3 . 


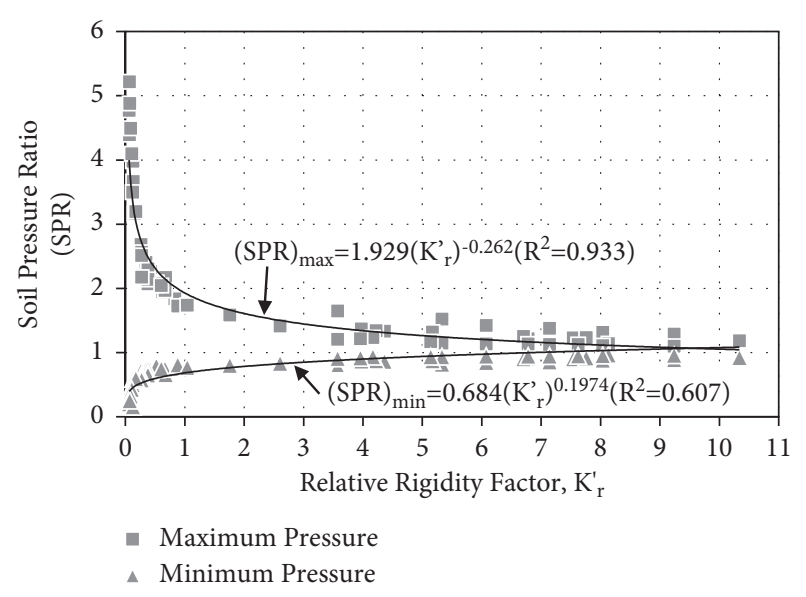

FIgURE 9: Relationship between soil pressure ratio and mat rigidity.

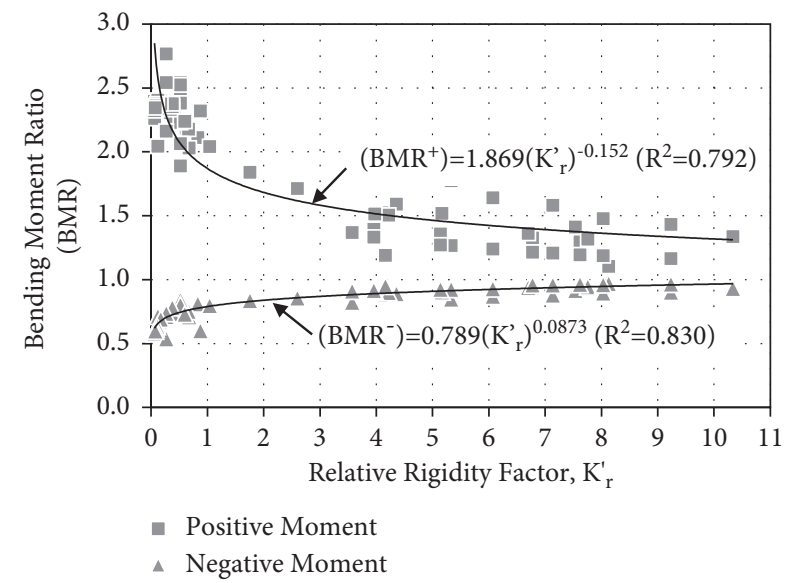

FIgURE 10: Relationship between bending moment ratio and mat rigidity.

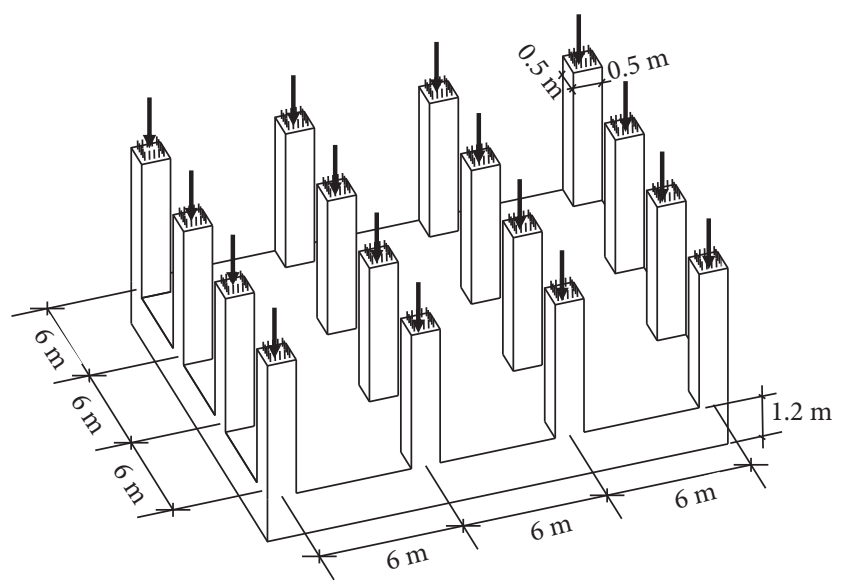

FIGURE 11: Geometry of the reference mat considered in the parametric analysis.

The procedure to developing sensitivity functions for the reference mat starts by considering a variable, such as the mat thickness, determining the relative rigidity factor from (3), and then substituting $K_{r}^{\prime}$ into the trend line equations
(4)-(7) to find approximate critical soil bearing pressure and bending moment ratios. The variable is then increased or decreased by a certain percentage and the procedure is repeated. Finally, the obtained $(\mathrm{SPR})_{\max },(\mathrm{SPR})_{\min }$, $(\mathrm{BMR}+)_{\max }$, and $(\mathrm{BMR}-)_{\max }$ values are plotted against the percentage change in the considered variable. For the reference mat shown in Figure 10, four variables are selected for the parametric study, namely, the modulus of elasticity of the concrete material, $E_{\mathrm{c}}$, mat thickness, $t$, soil modulus of subgrade reaction, $k_{\mathrm{s}}$, and the clear spacing between the columns, (L-l) and (B-b). These variables are changed in $10 \%$ increments within range of $-60 \%$ to $+60 \%$. Results of the parametric analysis are presented in Figure 12.

In general, the results in Figure 12 indicate that the mat thickness and clear span between columns are more critical than the modulus of elasticity of the concrete and modulus of subgrade reaction because the former two variables carry more weight in the rigidity equation. Furthermore, the maximum soil bearing pressure and maximum positive bending moment are more affected by the change in the variables than the minimum soil bearing pressure and maximum negative bending moment. Since the chosen reference mat has $K_{r}{ }_{r}=1$, which is defined in the paper as the threshold between rigid and flexible mat, an increase in $E_{\mathrm{c}}$ or $t$ and a decrease in $k_{\mathrm{s}}$ or (L-l) (B-b) increases $K_{r}^{\prime}$ above 1.0, leading to a rigid mat behavior. Conversely, a decrease in $E_{\mathrm{c}}$ or $t$ and an increase in $k_{\mathrm{s}}$ or (L-l) (B-b) decreases $K_{r}{ }_{r}$ below 1.0 , resulting in a flexible mat behavior. As expected, the variation in responses are more pronounced for relatively flexible mats than for relatively rigid mats because the trend lines within the range $K_{r}<1$ have steeper slopes than within the range $K_{r}>1$, as presented earlier in Figures 8 and 9. It should be noted that the results of the parametric study are only applicable for the reference mat under consideration. Any other mat having different geometry and material properties shall be analyzed with the same procedure.

\section{Application}

The symmetrical mat consisting of 3 bays in each perpendicular direction that has been used in the parametric study, and shown in Figure 11, is considered with some modifications. The spacing between columns is $6 \mathrm{~m}$, modulus of elasticity of concrete is $25 \mathrm{GPa}$, soil modulus of subgrade reaction is $50,000 \mathrm{kN} / \mathrm{m}^{3}$, and soil Poisson's ratio is 0.3 . Each of the 16 columns has square cross section with side equal to $0.3 \mathrm{~m}$. Load on corner columns is $250 \mathrm{kN}$, on the edge columns is $500 \mathrm{kN}$ and on the interior columns is $1000 \mathrm{kN}$, and the self-weight of the mat and any uniform live load directly applied on the top surface of the mat are ignored.

First, the mat is assumed to be infinitely rigid (mat thickness $=100.0 \mathrm{~m}$ ), leading to uniform soil bearing pressure equal to $q_{\text {rigid }}=27.8 \mathrm{kPa}$, obtained either by the SAFE software or by summing up all the column loads and dividing the result by the total mat area. Structural analysis of the mat foundation using the SAFE software in this case results in maximum positive and negative bending moments equal to $\left(M^{+}\right)_{\text {rigid }}=166.7 \mathrm{kN}-\mathrm{m} / \mathrm{m}$ and $\left(M^{-}\right)_{\text {rigid }}=395.0 \mathrm{kN}$ $\mathrm{m} / \mathrm{m}$, respectively. The corresponding maximum shear on a 


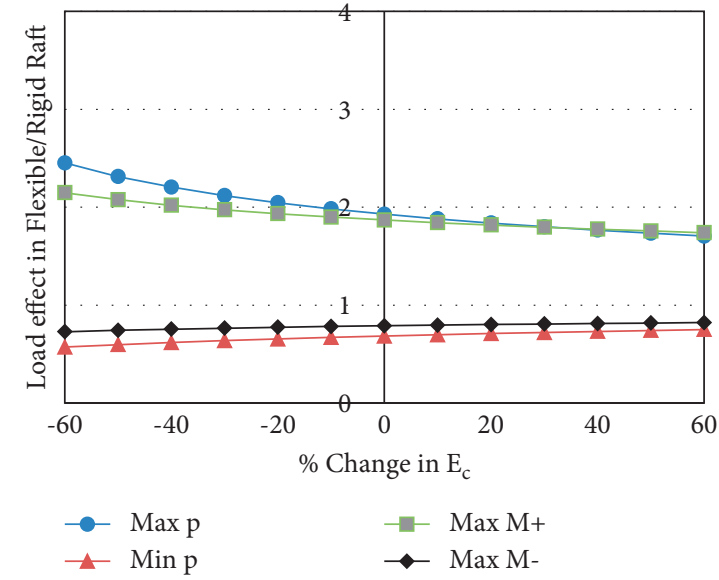

(a)

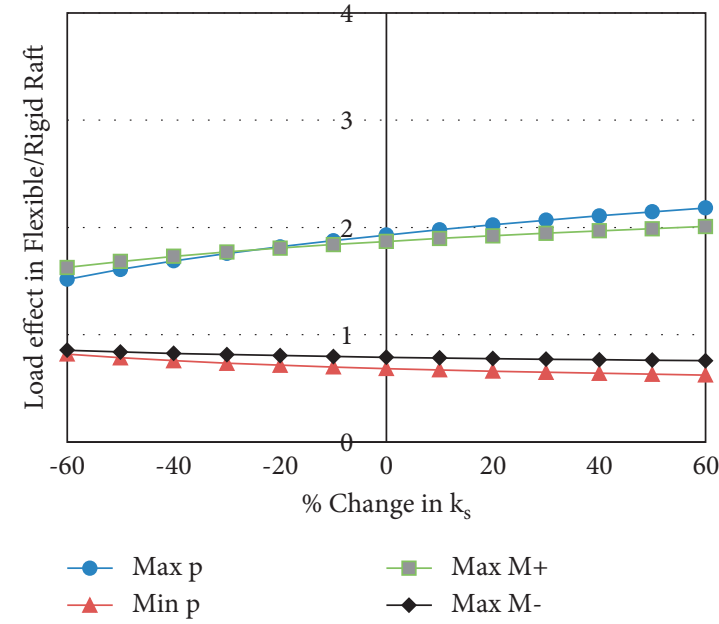

(c)

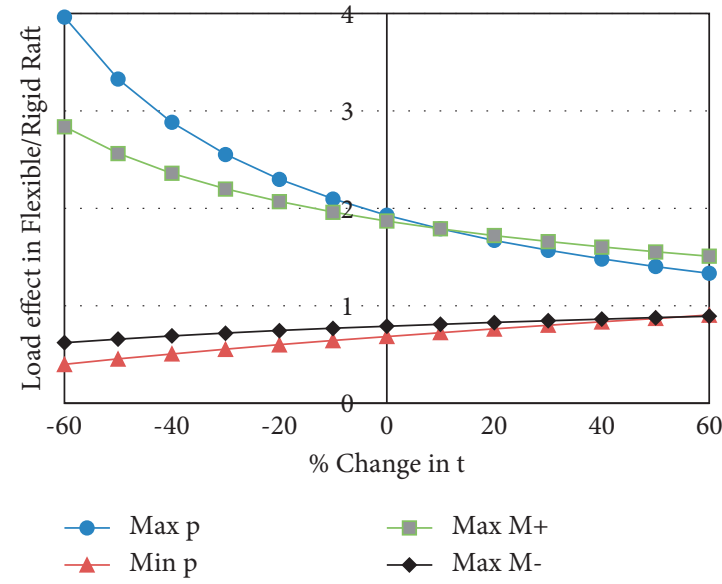

(b)

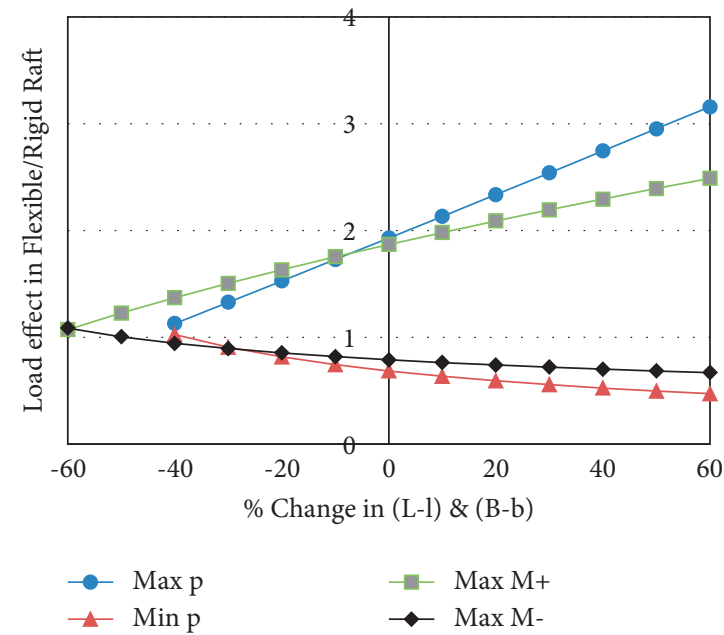

(d)

Figure 12: Effect of change in variables on mat response ratio. (a) Modulus of elasticity of concrete, $E_{c}$. (b) Thickness of the mat, $t$. (c) Modulus of subgrade reaction of soil, $k_{s}$. (d) Clear spacing between columns, $(L-1)(\mathrm{B}-\mathrm{b})$.

unit-wide strip of the mat is $V_{\text {rigid }}=527.5 \mathrm{kN} / \mathrm{m}$. In order to illustrate the practical implementation of the results of the study, the reference mat is then analyzed twice: once with a thickness equal to $1.0 \mathrm{~m}$ and another time with a thickness equal to $2.0 \mathrm{~m}$.

8.1.1.0 $\mathrm{m}$ Thick Mat. The relative rigidity factor for the mat is calculated using equation (3):

$$
\begin{aligned}
K_{r}^{\prime} & =\frac{E_{c} t^{3}}{k_{s}\left(1-\mu_{s}^{2}\right)(L-l)^{2}(B-b)^{2}} \\
& =\frac{25,000,000(1)^{3}}{50,000\left(1-0.3^{2}\right)(6-0.3)^{2}(6-0.3)^{2}} \\
& =0.520 .
\end{aligned}
$$

Using the power functions that relate the maximum and minimum soil bearing pressure ratios to the rigidity factor, equation (4) and (5), we get

$$
\begin{aligned}
(\mathrm{SPR})_{\max } & =1.929\left(K_{r}^{\prime}\right)^{-0.262} \\
& =1.929(0.52)^{-0.262} \\
& =2.290 .
\end{aligned}
$$

$$
(\mathrm{SPR})_{\min }=0.684\left(K_{r}^{\prime}\right)^{0.1974}=0.684(0.52)^{0.1974}=0.601 \text {. }
$$

The corresponding power functions that relate the maximum positive and negative bending moment ratios to the rigidity factor, obtained from equation (6) and (7), are

$$
\begin{aligned}
\left(B M R^{+}\right)_{\max } & =1.869\left(K_{r}^{\prime}\right)^{-0.152} \\
& =1.869(0.52)^{-0.152} \\
& =2.064 \\
\left(B M R^{-}\right)_{\max } & =0.789\left(K_{r}^{\prime}\right)^{0.0873} \\
& =0.789(0.52)^{0.0873} \\
& =0.746 .
\end{aligned}
$$


TABLE 2: Comparison of predicted and FEA results for the considered mats.

\begin{tabular}{|c|c|c|c|c|c|c|c|c|}
\hline \multirow{2}{*}{ Mat } & \multirow{2}{*}{$t(\mathrm{~m})$} & \multirow{2}{*}{$K_{r}^{\prime}$} & \multicolumn{3}{|c|}{$q_{\max }$} & \multicolumn{3}{|c|}{$q_{\min }$} \\
\hline & & & Predicted & FEA & Error & Predicted & FEA & Error \\
\hline Flexible & 1 & 0.52 & 63.7 & 58.5 & $8.9 \%$ & 16.7 & 20.5 & $18.5 \%$ \\
\hline Moderately rigid & 2 & 4.16 & 36.9 & 37.2 & $0.8 \%$ & 25.2 & 24.2 & $4.1 \%$ \\
\hline \multirow{2}{*}{ Mat } & \multirow{2}{*}{$t(\mathrm{~m})$} & \multirow{2}{*}{$K_{r}^{\prime}$} & \multicolumn{3}{|c|}{$\left(M^{+}\right)_{\max }$} & \multicolumn{3}{|c|}{$\left(M^{-}\right)_{\max }$} \\
\hline & & & Predicted & FEA & Error & Predicted & FEA & Error \\
\hline Flexible & 1 & 0.52 & 344 & 370 & $7.0 \%$ & 294.7 & 307.5 & $4.2 \%$ \\
\hline Moderately rigid & 2 & 4.16 & 250.9 & 252.5 & $0.6 \%$ & 353.1 & 350 & $0.9 \%$ \\
\hline
\end{tabular}

Using the above values, one can estimate the maximum and minimum soil bearing pressures as well as the maximum positive and negative bending moments in the $1.0 \mathrm{~m}$ thick mat without using structural analysis:

$$
\begin{aligned}
q_{\max } & =(\mathrm{SPR})_{\max }\left(q_{\text {rigid }}\right) \\
& =2.290 \times 27.8 \\
& =63.7 \mathrm{kPa}, \\
q_{\min } & =(\mathrm{SPR})_{\min }\left(q_{\text {rigid }}\right) \\
& =0.601 \times 27.8 \\
& =16.7 \mathrm{kPa}, \\
M^{+} & =\left(B M R^{+}\right)_{\max }\left(M^{+}\right)_{\text {rigid }} \\
& =2.064 \times 166.7 \\
& =344.0 \mathrm{kN}-\mathrm{m} / \mathrm{m}, \\
M^{-} & =\left(B M R^{-}\right)_{\max }\left(M^{-}\right)_{\text {rigid }} \\
& =0.746 \times 395 \\
& =294.7 \mathrm{kN}-\mathrm{m} / \mathrm{m} .
\end{aligned}
$$

8.2. $2.0 \mathrm{~m}$ Thick Mat. The rigidity factor for the $2.0 \mathrm{~m}$ thick mat is

$$
\begin{aligned}
K_{r}^{\prime} & =\frac{E_{c} t^{3}}{k_{s}\left(1-\mu_{s}^{2}\right)(L-l)^{2}(B-b)^{2}} \\
& =\frac{25,000,000(2)^{3}}{50,000\left(1-0.3^{2}\right)(6-0.3)^{2}(6-0.3)^{2}} \\
& =4.16 .
\end{aligned}
$$

Using the power functions for the maximum and minimum soil bearing pressure ratios, we get

$$
\begin{aligned}
(\mathrm{SPR})_{\max } & =1.929\left(K_{r}^{\prime}\right)^{-0.262} \\
& =1.929(4.16)^{-0.262} \\
& =1.327 .
\end{aligned}
$$

$(\mathrm{SPR})_{\min }=0.684\left(K_{r}^{\prime}\right)^{0.1974}=0.684(4.16)^{0.1974}=0.906$.

Utilizing the corresponding power functions for the maximum positive and negative bending moment ratios, we obtain

$$
\begin{aligned}
\left(B M R^{+}\right)_{\max } & =1.869\left(K_{r}^{\prime}\right)^{-0.152} \\
& =1.869(4.16)^{-0.152} \\
& =1.505 \\
\left(B M R^{-}\right)_{\max } & =0.789\left(K_{r}^{\prime}\right)^{0.0873} \\
& =0.789(4.16)^{0.0873} \\
& =0.894 .
\end{aligned}
$$

Using the above ratios, the maximum and minimum soil bearing pressures and the maximum positive and negative bending moments in the $2 \mathrm{~m}$ thick mat can be predicted:

$$
\begin{aligned}
q_{\max } & =(\mathrm{SPR})_{\max }\left(q_{\text {rigid }}\right) \\
& =1.327 \times 27.8 \\
& =36.9 \mathrm{kPa}, \\
q_{\text {min }} & =(\mathrm{SPR})_{\min }\left(q_{\text {rigid }}\right) \\
& =0.906 \times 27.8 \\
& =25.2 \mathrm{kPa}, \\
M^{+} & =\left(B M R^{+}\right)_{\max }\left(M^{+}\right)_{\text {rigid }} \\
& =1.505 \times 166.7 \\
& =250.9 \mathrm{kN}-m / m, \\
M^{-} & =\left(B M R^{-}\right)_{\max }\left(M^{-}\right)_{\text {rigid }} \\
& =0.894 \times 395 \\
& =353.1 \mathrm{kN}-m / m .
\end{aligned}
$$

To evaluate the accuracy of the estimated critical soil bearing pressures and internal bending moments of the nonrigid mats, we compare them to the finite element results, which consider the actual mat thickness and soil flexibility. Table 2 compares the critical soil bearing pressures and internal bending moments for the flexible $(1.0 \mathrm{~m}$ thick) and moderately rigid ( $2.0 \mathrm{~m}$ thick) mats to the corresponding values from the finite element analysis, respectively. In general, the power equations can predict the response of the moderately rigid mat more accurately than the flexible mat. Aside from the minimum soil pressure, the error in the predicted results is less than $10 \%$. While the error in the predicted minimum soil pressure for the flexible mat seems high (18.5\%), such an action in practice is only important for checking the potential of uplift under the mat and has little impact on structural design of the mat. 


\section{Summary and Conclusions}

This study researches the relationship between mat rigidity and response in terms of the critical soil bearing pressure, bending moment, and shear. The finite element analysis method is used to model a large number of regular and irregular mats subjected to concentrated loads. The mat was modeled within the linearly elastic range by shell elements and the soil by elastic springs lumped the location of the nodes. The parameters that are varied in the study are the mat thickness, spacing between columns, modulus of elasticity of concrete, soil modulus of subgrade reaction, and column cross section dimensions. Results of the study lead to the following conclusions:

(1) The maximum and minimum soil bearing pressures as well as the maximum positive and negative bending moments within the mat are highly dependent on the rigidity of the mat. Therefore, using the rigid mat analysis procedure to predict the response of a flexible mat can lead to erroneous results.

(2) The critical shear force in the mat is independent of the mat rigidity; hence, it can be predicted by analyzing an equivalent rigid mat with the same geometry and properties.

(3) A dimensionless rigidity factor, $K_{r}^{\prime}$, is developed that quantifies the stiffness as a function of mat thickness, modulus of elasticity of the concrete, soil subgrade reaction, soil Poisson's ratio, maximum spacing between columns along the two major axes of the mat, and column cross section dimensions.

(4) The relationships between the developed mat rigidity factor and the normalized mat response ratios showed that a threshold $K_{r}^{\prime}=1$ defines the boundary between a flexible and rigid mat. The response of a flexible mat having $K_{r}^{\prime}<1$ cannot be structurally analyzed as a rigid mat due to the differences in the critical soil bearing pressures and internal positive and negative bending moments.

(5) Power functions are developed from the finite element results that relate the mat rigidity to the critical soil bearing pressures and bending moments with reasonable accuracy, as demonstrated by the high coefficients of determination.

(6) The impact of mat thickness and clear span between columns on the response of the raft are more critical than the modulus of elasticity of the concrete and modulus of subgrade reaction. Furthermore, the maximum soil bearing pressure and maximum positive bending moment are more affected by the change in the variables than the minimum soil bearing pressure and maximum negative bending moment.

\section{Data Availability}

The data used to support the findings of this study are available from the corresponding author upon request.

\section{Conflicts of Interest}

The authors declare that there are no conflicts of interest regarding the publication of this paper.

\section{Acknowledgments}

Special thanks are due to former graduate student Mr. Pouya Partazian for helping out with the finite element analyses. The authors would like to recognize the financial support provided by the Office of Research at the American University of Sharjah, UAE, through Faculty Research Grant FRG11-II-22.

\section{References}

[1] J. E. Bowles, Foundation Analysis and Design, McGraw-Hill, NY, USA, 5th edition, 1997.

[2] ACI-336.2R-88, Suggested Analysis and Design Procedures for Combined Footings and Mats, American Concrete Institute, MI, USA, 2002.

[3] DIN. 4018, Berechnung der Sohldruckverteilung unter Flächengründungen, Beuth Verlag GmbH, Berlin, Germany, 1981.

[4] K. Ueshita and G. G. . Meyerhof, "Surface displacements of an elastic layer under uniformly distributed loads," Highway Research Record, vol. 228, pp. 1-10, 1971.

[5] D. M. Milovic and J. P. Tournier, "Stresses and displacements due to rectangular foundation on a layer of finite thickness," Soils and Foundations, vol. 13, no. 4, pp. 29-43, 1973.

[6] H. D. Tabakman and A. H. Hadjian, "Evaluation of nuclear power plant structure foundation rigidities," ASCE Specialty Conference on Structural Design of Nuclear Plant Facilities, vol. 8-10, pp. 739-762, 1975.

[7] B. Ramanathan, "New concept for the relative rigidity of rafts," in Proceedings of the International Conference on Numerical Methods in Geomechanics, vol. 1, pp. 489-495, ASCE, VI, USA, 1976.

[8] B. Ramanathan and K. L. Pujar, "Effect of superstructure rigidity in raft design," in Proceedings of the International Symposium on Soil Structure Interaction, pp. 117-121, Abhay Rastogi for Sarita Prakashan, Uttarkhand, India, January 1977.

[9] B. L. Mehrotra, Y. P. Gupta, A. K. Baska, and A. K. Govil, "Approximate method - raft-structure interaction analysis," in Proceedings of the Annual Conference Can Soc for Civ Eng, Univ of Manit, Winnipeg, p. 12, Bhopal, India, May 1980.

[10] G. Waas and H. R. Riggs, "Effect of the flexibility of the base mat on seismic response of a PWR-reactor building," in Proceedings of the 7th International Conference on Structural Mechanics in Reactor Technology, Chicago, Illinois, August 1983.

[11] H. R. Riggs and G. Waas, "Influence of foundation flexibility on soil-structure interaction," Earthquake Engineering \& Structural Dynamics, vol. 13, no. 5, pp. 597-615, 1985.

[12] Z. E. Yao and J. R. Zhang, "Assessment of the effects of structure/raft/soil interaction," in Proceedings of the 5th international conference on Numerical Methods in Geomechanics, Japan Soc of Civil Engineers, pp. 813-819, A. A. Balkema, Nagoya University, Nagoya, Japan, April 1985.

[13] Y. K. Chow, "Vertical deformation of rigid foundations of arbitrary shape on layered soil media," International Journal 
for Numerical and Analytical Methods in Geomechanics, vol. 11, no. 1, pp. 1-15, 1987.

[14] K. Zilch, "Soil - structure interaction," in Proceedings of the safety and performance concepts: contributions to the workshop-sessions "model uncertainties", "new concepts" and "full scale testing", organized by task groups of commission 1, pp. 73-98, Comité Euro-International du Béton (CEB), London, United Kingdom, September 1993.

[15] K. Horikoshi and M. F. Randolph, "On the definition of raftsoil stiffness ratio for rectangular rafts," Géotechnique, October, vol. 47, no. 5, pp. 1055-1061, 1997.

[16] J. A. Hemsley, Elastic Analysis of Raft Foundations, p. 663, ICE Publishing: Thomas Telford, Technology \& Engineering, London, UK, 1998.

[17] A. Sutradhar, "An improved design rationale for mat foundation based on finite element analysis," A Thesis Submitted in Partial Fulfilment of the Requirements for the Degree of Master of Science in Civil Engineering, Thesis, Bangladesh University of Engineering and Technology, Dhaka, Bangladesh, 1999.

[18] R. C. Chown and M. S. Crilly, "Performance of a semi-rigid raft foundation on soft ground under vertical load," Ground Engineering, EMAP Business Communications, vol. 33, no. 3, pp. 36-40, 2000.

[19] J. F. Gong, X. L. Huang, and J. Teng, "Rigidity characteristic and deformation calculation of large-area thick raft foundation," in Proceedings of the 16th International Conference on Soil Mechanics and Geotechnical Engineering: Geotechnology in Harmony with the Global Environment, vol. 3, pp. 1471-1475, Millpress Science Publishers, Osaka, Japan, September 2005.

[20] L. Edgers, M. Sanayei, and J. Alonge, "Modeling the effects of soil-structure interaction on a tall building bearing on a mat foundation," in Civil Engineering Practicevol. 20, no. 2, pp. 51-68, Boston Society of Civil Engineers Section, 2005.

[21] N. O. Farag, K. M. E. Zahaby, and A. S. Bazaraa, "Quantifying soil-raft-superstructure interaction using field testing, numerical analysis and artificial neural networks, ANNs," in Proceedings of the Structures Congress and Exposition, American Society of Civil Engineers, Structural Engineering; Institute (SEI), pp. 945-956, NY, USA, April 2005.

[22] D. D. Thangaraj and K. Ilamparuthi, "Parametric study on the performance of raft foundation with interaction of frame," Electronic Journal of Geotechnical Engineering, vol. $15 \mathrm{H}$, pp. 1-18, 2010.

[23] A. E. Arapakou and V. P. Papadopoulos, "Factors Affecting Differential Settlements of Framed Structures," Geotechnical and Geological Engineering, Springer Netherlands, vol. 30, no. 6, pp. 1323-1333, 2012.

[24] J. R. Omer and A. Arbabi, "Evaluation of Finite Element, Finite Difference and Elasticity Methods for Hypothetical Raft Foundations Installed on Layered Strata," Geotechnical and Geological Engineering, vol. 33, no. 4, pp. 1129-1140, 2015.

[25] D. Lątka and M. Repelewicz, "The Influence Of Superstructure Stiffness On Internal Forces Distribution In Raft Foundations," Technical Transactions - Civil Engineering, vol. 4-B, no. 28, pp. 51-56, 2015.

[26] M. B. Venkatesh and R. D. Deshpande, "Analysis of R.C. building frame with raft foundation considering soil-structure interaction," International Research Journal of Engineering and Technology, vol. 04, no. 05, pp. 752-760, 2017.

[27] S. Tabsh and M. E. Emam, "Finite element-based parametric analysis of mat foundations," in Proceedings of the 8th European Conference on Numerical Methods in Geotechnical
Engineering, vol. 1, pp. 693-697, A.A. Balkema, Delft, Netherlands, June 2014.

[28] S. W. Tabsh, M. E. Emam, and P. Partazian, "Numerically based parametric analysis of mat foundations," Practice Periodical on Structural Design and Construction, vol. 25, no. 2, Article ID 04020009, 2020.

[29] CSI Analysis Reference Manual for SAP2000, ETABS, SAFE and CSiBridge, p. 534, Computers and Structures, Inc., Berkeley, CA, 2016.

[30] SAFE Verification Manual, Computers and Structures, p. 505, Inc. (CSI), Berkeley, CA, 2016.

[31] G. G. Meyerhof, "Generalbericht: Soil-Structure Interaction and Foundations," in Proceedings of the 6th Pan-American Soil Mechanics and Foundation Engineering Congress, Lima, Peru, December, 1979.

[32] A. S. Vesic, "Bending of beams resting on isotropic elastic solid," Journal of the Engineering Mechanics Division, vol. 87, no. 2, pp. 35-53, 1961.

[33] A. S. Vesic, "Beams on elastic subgrade and the Winkler's hypothesis," in Proceedings of the 5th Int. Conf. on Soil Mechanics and Foundation Engineering, pp. 845-850, Paris, France, July 1961.

[34] S. W. Tabsh and A. Raouf Al-Shawa, "Effect of spread footing flexibility on structural Response," Practice Periodical on Structural Design and Construction, vol. 10, no. 2, pp. 109-114, 2005. 